Disponible en ligne :

Données d'enquêtes socioéconomiques sur les ménages agricoles dans les pays du Sud. Coordinatrices: Sandrine Freguin-Gresh, Céline Dutilly www.cahiersagricultures.fr

\title{
Explorer les liens entre agriculture et sécurité alimentaire: une enquête auprès des femmes du gouvernorat de Sidi-Bouzid en Tunisie
}

\author{
Cédric Gaillard ${ }^{1,2, *}$, Sofyan Martin ${ }^{5}$, Pierre-Marie Bosc ${ }^{1,2}$, Jalila El-Ati ${ }^{3}$, Marie Claude Dop ${ }^{4}$, \\ Tarek Trabelsi ${ }^{3}$, Marie-Josephe Amiot ${ }^{2,6}$ et Sandrine Dury ${ }^{1,2}$ \\ ${ }^{1}$ CIRAD, UMR MOISA, 34398 Montpellier, France \\ 2 MOISA, Univ Montpellier, Montpellier, France \\ ${ }^{3}$ INNTA (Institut national de nutrition et de technologie alimentaire), Laboratoire SURVEN (Surveillance et épidémiologie \\ nutritionnelle), Tunis, Tunisie \\ ${ }^{4}$ UMR NUTRIPASS, Institut de Recherche pour le développement, Université de Montpellier, SupAgro, Montpellier, France \\ ${ }^{5}$ EGIS International, Paris, France \\ ${ }^{6}$ INRA, UMR MOISA, Montpellier, France
}

\begin{abstract}
Résumé - Cet article présente et décrit le contenu d'une base de données construite à partir d'une enquête menée dans le gouvernorat de Sidi-Bouzid, situé dans le centre de la Tunisie. Sur ce territoire en transition, le secteur agricole, qui tient une place prédominante dans l'activité économique, est caractérisé par la diversité de ses exploitations, aussi bien sur le plan organisationnel (main-d'œuvre familiale ou salariée à dominante féminine) que technique (présence d'infrastructure d'irrigation, de culture sous-abri). Sur le plan des orientations technico-économiques, les cultures arboricoles et maraîchères se développent quand d'autres cultures plus traditionnelles persistent (oléiculture, céréaliculture). Des politiques publiques récentes ont également encouragé l'élevage laitier. Berceau de la révolution du Jasmin en 2010, Sidi-Bouzid fait face à des difficultés économiques et sociales. Dans ce contexte, l'agriculture a un rôle essentiel pour assurer la sécurité alimentaire de la population. L'objectif de cette enquête était d'identifier les liens existants entre la structure, le fonctionnement et les performances des exploitations agricoles, et la sécurité alimentaire des femmes adultes du territoire. Les revenus individuels des membres du ménage, l'autoconsommation de produits agricoles, et l'autonomie des femmes peuvent aussi jalonner ce chemin d'impact. En effet, le développement du salariat peut permettre aux femmes d'accroître leur autonomie, privilégiant ainsi, à travers la maîtrise de leurs dépenses, leurs besoins alimentaires et ceux du ménage auquel elles appartiennent. L'enquête est articulée autour de trois questionnaires. Cinq cent soixante-quinze femmes, sélectionnées aléatoirement à partir d'un échantillon représentatif des femmes de 20 à 49 ans du gouvernorat de Sidi-Bouzid, 575 chefs de ménages et 316 chefs d'exploitations ont répondu aux questionnaires. La collecte a été réalisée sur questionnaire papier, en entretien face-à-face en décembre 2015. Les données ont été ensuite saisies et contrôlées sur le logiciel EpiData, avant d'être nettoyées sur le logiciel Stata. Les données sont disponibles sur le lien doi: 10.18167/DVN1/LWT7BG.
\end{abstract}

Mots clés : sécurité alimentaire / femme / autonomisation / dépense des ménages / exploitation agricole familiale

Abstract - Exploring the links between agriculture and food security: a survey of women in the governorate of Sidi-Bouzid, central Tunisia. This paper presents and describes the contents of a database built from a survey conducted in central Tunisia in the governorate of Sidi-Bouzid. In this evolving region, the agricultural sector, which occupies a predominant place in economic activity, is characterized by the diversity of its farms, both at the organizational level (family or salaried workforce predominantly female) and technical (presence of irrigation infrastructure, under-cover cultivation). In terms of technical and economic orientations, tree crops and market gardening develop when other more traditional crops persist

\footnotetext{
*Auteur de correspondance : cedric.gaillard@cirad.fr
} 
(olive cultivation, cereal farming). Recent public policies have also encouraged dairy farming. Cradle of the Jasmin revolution in 2010, Sidi-Bouzid faces economic and social difficulties. In this context, agriculture has a key role in ensuring food security for population. The objective of this survey was to identify the links between the structure, functioning and performance of farms and the food security of adult women in the territory. Individual incomes of household members, self-consumption of agricultural products, and women empowerment may also punctuate this impact pathway. Indeed, women's income could increase their autonomy, thus favoring, through the control of their expenditure, their food needs and those of the household to which they belong. The survey is based on three questionnaires. A sample of 575 women, selected from a representative sample of 20 to 49 -year-old women from the governorate of Sidi-Bouzid, 575 heads of households and 316 heads of farms responded to the questionnaires. The collection was carried out on a paper questionnaire in face-to-face interview in December 2015. The data were then entered and checked on the EpiData software before being exported and cleaned on the Stata software. The data are available in following the link doi: 10.18167/DVN1/LWT7BG.

Keywords: food security / women / empowerment / expenditure households / family farms

\section{Cadre conceptuel de l'enquête}

$\mathrm{Au}$ sein des études sur la durabilité des systèmes alimentaires, on retrouve une série de travaux plus ou moins récents qui s'intéressent aux relations entre la production agricole et la nutrition (Hoddinott, 2012; Gomez et al., 2013). Les cadres conceptuels illustrant ces relations (Gillespie et Kadiyala, 2012 ; Haddad, 2000 ; Herforth et al., 2015) séparent les chemins d'impact au niveau «ménage» ou «inframénage» agricole des chemins d'impact qui relient les producteurs aux consommateurs, via les flux de produits, les marchés (de produits et/ou de travail) et via l'environnement. $\mathrm{Au}$ niveau des ménages producteurs (ou exploitations familiales), la production agricole a des liens directs avec la nutrition via l'autoconsommation, ou des liens indirects via les revenus agricoles (Carletto et al., 2015). D'autres liens indirects, souvent rassemblés sous la bannière de «women empowerment» (Alkire et al., 2013), associés aux droits des femmes et à leur capacité à avoir un revenu propre et à pouvoir l'utiliser, ont un effet significatif sur leur nutrition (Malapit et al., 2015). Les différents modèles conceptuels cités se basent tous sur le modèle causal qui suppose que la malnutrition dépend de facteurs individuels: santé et alimentation, et parfois soins (Martin-Prével, 2002). Ces facteurs sont reliés entre eux et dépendent d'autres facteurs sous-jacents au niveau du ménage, en particulier du niveau de vie qui détermine la sécurité alimentaire (accès régulier à des aliments sains suffisants...).

Contrairement à ce qui est habituellement réalisé, nous nous intéressons ici à la sécurité alimentaire des individus (les femmes) et recherchons les corrélations avec d'autres facteurs, en particulier agricoles.

Ainsi, nous faisons l'hypothèse que l'agriculture influence la sécurité alimentaire des femmes à travers deux chemins d'impact principaux :

- les revenus féminins salariés et le gain d'autonomie qui y est associé (Gillespie et al., 2012);

- l'accès direct à des produits de l'exploitation familiale permettant une alimentation de meilleure qualité (Sibhatu et al., 2015).

Le questionnaire a été bâti pour tester ces hypothèses et déterminer, d'une part, quelle était la perception des femmes de leur propre sécurité alimentaire à travers un questionnaire normé s'inspirant du HFIAS (Household Food Insecurity Access Scale (Coates et al., 2007), développé par l'United States Agency for International Development) et, d'autre part, le statut et le rôle de ces femmes au sein du ménage, qu'elles soient salariée agricole, travailleuse indépendante, salariée dans une autre activité ou femme au foyer. Pour ce dernier point, des variables mesurant le niveau d'activité des femmes, leur autonomie et leur pouvoir de décision ont été mobilisées.

Le questionnaire HFIAS est composé de 9 questions permettant de mesurer l'incertitude ou l'angoisse des ménages vis-à-vis de la quantité ou de la qualité de la nourriture consommée durant une période de 4 semaines précédant l'enquête. Ici, la mesure se fait au niveau individuel (auprès des femmes, on nommera ce questionnaire IFIAS pour Individual Food Insecurity Access Scale) et sur une période de 2 mois précédant l'enquête.

Enfin, de nombreuses variables permettant de caractériser les exploitations agricoles (nature, diversité, structure, performance) et les préférences de consommation des ménages (à travers leurs dépenses), participent à une meilleure compréhension de l'environnement auquel la femme est liée.

Cette enquête fait suite à une autre enquête réalisée auprès de la même population, visant à déterminer la consommation alimentaire des femmes de la région.

\section{Description des données}

\subsection{Unité d'observation}

Les problématiques présentées en introduction nous ont conduits à structurer le questionnaire en trois volets correspondant à trois unités d'observations reliées (Fig. 1):

- la femme, interrogée en premier, qui permet d'identifier le chef du ménage dans lequel elle vit et le chef d'exploitation dans le cas où elle vit ou travaille sur une exploitation agricole;

- le ménage, défini comme le groupe qui partage un seul et même budget. Le chef de ménage identifié puis interrogé est le gestionnaire des dépenses du ménage;

- dans le cas des ménages agricoles, l'exploitation agricole familiale. Le chef d'exploitation identifié puis interrogé est le gestionnaire principal de l'exploitation. 


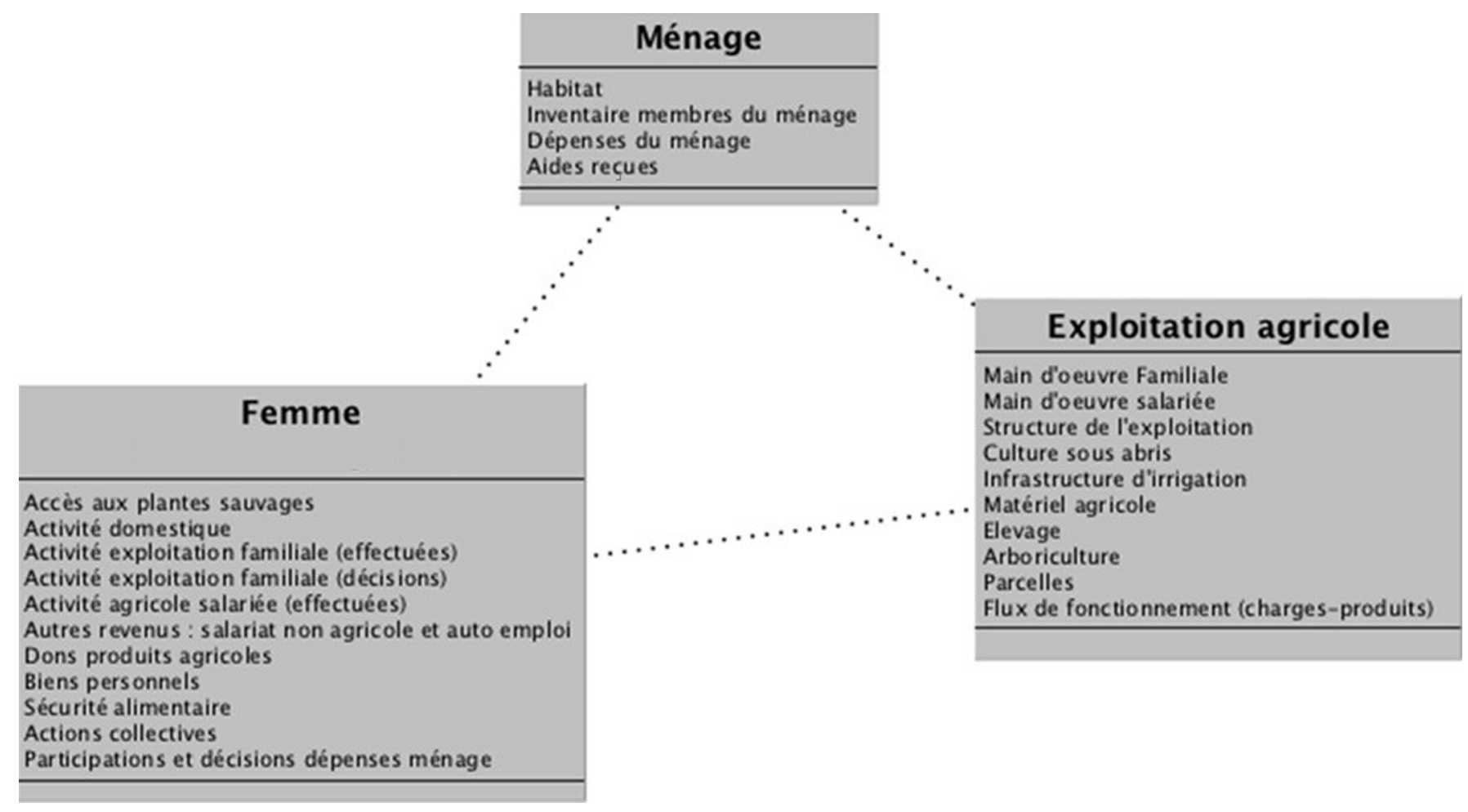

Fig. 1. Unités d'observation et rubriques correspondantes. Source: Martin S., 2016.

Fig. 1. Observation units and corresponding sections. Source: Martin S., 2016.

Il arrive qu'à défaut du chef de ménage ou du chef d'exploitation, la personne interrogée soit la personne disponible permettant d'obtenir le plus d'information sur ces unités d'observation. Les questions relatives au volet «ménage » et au volet «exploitation agricole» sont essentiellement formelles et/ou quantitatives, limitant ainsi les biais potentiels dans le cas où la personne interrogée est différente $\mathrm{du}$ «représentant légal» de ces deux unités d'observation.

\subsection{Spécificités du terrain d'étude}

Le gouvernorat du centre de la Tunisie, Sidi Bouzid (Fig. 2), abrite en 2014 une population de 429912 habitants, à majorité rurale $(73 \%)$ (Institut national de la statistique. Recensement général de la population et de l'habitat, 2014). Administrativement, le gouvernorat est découpé en douze délégations (en Tunisie, une délégation est une circonscription administrative intermédiaire entre le gouvernorat et le secteur ou district). Malgré un climat aride à semi-aride et une pluviométrie faible et irrégulière, le gouvernorat constitue l'un des pôles agricoles tunisiens, grâce notamment à des potentialités hydriques souterraines favorables.

Sur les plaines fertiles de la région, on retrouve des cultures maraîchères, céréalières, fourragères et arboricoles. Depuis quelques années, le territoire connaît une transition de son modèle agricole, passant d'un système traditionnel homogène de producteurs pastoraux dont l'alimentation reposait sur l'autoconsommation, à un système mixte agro-pastoral plus

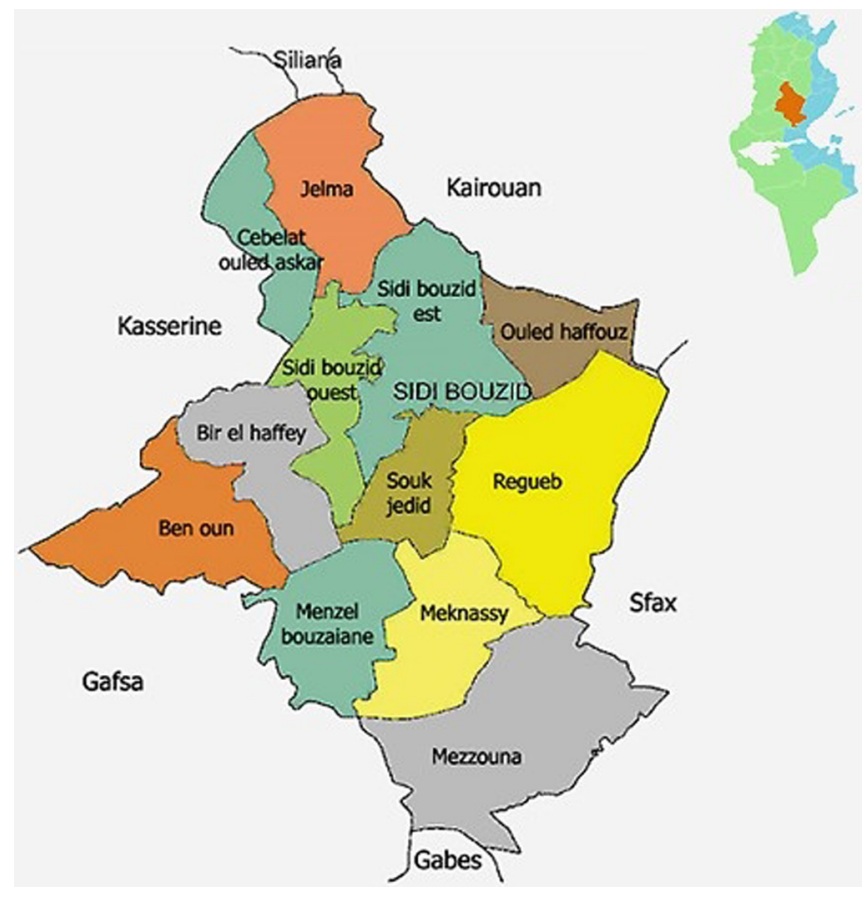

Fig. 2. Gouvernorat de Sidi-Bouzid. Source: ministère de l'Agriculture, des Ressources hydrauliques et de la Pêche de Tunisie.

Fig. 2. Governorate of Sidi-Bouzid. Source: Ministry of Agriculture, Water Resources and Fisheries of Tunisia. 
productif, mais où l'alimentation des ménages est plus dépendante du marché (Ferchiou, 2017).

Si près d'un tiers des actifs travaille dans l'agriculture, ce dynamisme reste tout relatif au regard de la situation socioéconomique du gouvernorat. Marqué par un taux de chômage élevé, la ville de Sidi-Bouzid, chef-lieu du gouvernorat, a constitué le point de tension initial de la révolution du Jasmin à la fin de l'année 2010.

À travers sa situation économique et sociale et la place importante du secteur agricole, cette région présente un intérêt particulier pour étudier les relations entre la sécurité alimentaire des populations et leurs moyens d'existence, appréhendés au niveau des ménages.

\subsection{Couverture temporelle}

La collecte d'informations a eu lieu entre le 20 décembre 2015 et le 21 janvier 2016.

L'enquête fait référence à la situation des femmes, des ménages et des exploitations agricoles interrogées fin 2015. Les différentes ressources, la production agricole et les dépenses des ménages sont indiquées pour l'année 2015.

La saisonnalité n'est donc pas abordée ici. Cependant, les femmes répondantes à l'IFIAS, qui mesure la perception qu'elles ont de leur propre sécurité alimentaire sur une période de 2 mois précédant l'enquête, peuvent être soumises à ces effets saisonniers.

\subsection{Population couverte par l'étude}

Les femmes, dont la contribution à la production agricole est souvent sous-estimée par les statistiques nationales tunisiennes (CREDIF, 2003 ; Gasmi, 2003 ; FAO AQUASTAT, 2014 ; Latreille, 2008), ont une place centrale dans l'étude.

Ainsi, l'entrée principale de l'étude est la femme âgée de 20 à 49 ans. Elle est appréhendée dans son environnement familial (ménage) et dans ses relations potentielles avec le secteur agricole, qu'elle vive et travaille sur une exploitation agricole familiale dont elle est membre, ou qu'elle travaille comme salariée dans une autre exploitation sans référence à un lien familial.

\section{Méthodologie}

\subsection{Collecteur des données}

Institut national de nutrition et de technologie alimentaire (INNTA).

\subsection{Autres contributeurs}

Au sein du groupe projet MEDINA, les nutritionnistes de l'UMR NUTRIPASS de l'Institut de la recherche pour le développement (IRD) ont eu un rôle contributeur particulier. Ils ont en effet conduit avec l'INNTA les 4 premiers passages de l'enquête nutritionnelle en s'appuyant sur la technique du rappel des 24 heures et celle du questionnaire de fréquence de consommation des produits de la biodiversité. Le $5^{\mathrm{e}}$ passage de l'enquête correspond à l'objet de la présente publication.

\subsection{Producteur}

Projet MEDINA (ANR-12-TMED-0004), «Promoting sustainable Mediterranean food systems for good nutrition and health », retenu dans le cadre du programme d'études transdisciplinaires sur la Méditerranée (TRANSMED).

\subsection{Méthode d'échantillonnage}

La conception du plan d'échantillonnage a été réalisée par l'INS (Institut national de la statistique) tunisien. Conçu pour répondre aux besoins des enquêtes nutritionnelles qui précédaient notre enquête, l'échantillonnage en grappes est destiné à être représentatif des femmes issues de la population âgée de 20 à 49 ans de Sidi-Bouzid (INS, 2014).

Pour étudier la population de Sidi-Bouzid d'un point de vue nutritionnel, la variable d'étude choisie pour calculer la taille de l'échantillon est l'apport calorique moyen journalier par adulte. En fixant une précision égale à $2 \%$, un risque d'erreur de première espèce $\alpha=5 \%$, un effet grappe égal à 1,5 et un taux de refus de 5\% (ces valeurs sont notamment préconisées par l'International Fund for Agricultural Development), le nombre de femmes requis était de 540. L'échantillon a ensuite été renforcé pour les délégations à forte concentration agricole et rurale. Le nombre de femmes a donc été porté à 720 .

L'unité de sondage étant le ménage, il a été fait le choix d'interroger une femme par ménage pour élargir la représentativité de la population de Sidi Bouzid. Ces 720 ménages ont été tirés au sort par sondage aléatoire stratifié à deux degrés :

- au premier degré, 36 districts ont été tirés parmi les 12 délégations de Sidi Bouzid. Leur nombre par délégation est proportionnel au nombre de ménages qu'ils représentent sur le gouvernorat;

- au second degré, 20 ménages ont été tirés au sort dans chaque district.

Avec 20 ménages par grappe, les effets de conception (surcroît de variance de l'échantillonnage en grappes par rapport à un échantillonnage simple de même taille) sont minimisés (ONU, 2010).

Dans chaque ménage, une femme âgée de 20 à 49 ans a été sélectionnée par tirage aléatoire parmi les femmes du même ménage et de la même tranche d'âge.

Les districts ont ensuite été classés en zone urbaine (ou zone communale telle qu'érigée par décret du ministère de l'Intérieur tunisien, souvent les communes de plus de 2000 habitants) et en zone rurale (ou zone non communale).

Le premier passage de l'enquête nutritionnelle a permis d'interroger 718 femmes (soit un taux de réponse proche de $100 \%$ ). Le cinquième passage, objet de la présente publication, a permis d'interroger 575 femmes (Tab. 1), soit un taux de réponse de plus de $80 \%$. Parmi les 143 cas de non-réponses par rapport à l'échantillon initial, 53 étaient absentes du domicile malgré les passages répétés des enquêtrices, 47 ont refusé d'être interrogées, 1 était hospitalisée et 42 ont déménagé.

Ce choix méthodologique quant à l'élaboration de l'échantillonnage est imparfait pour les besoins de l'enquête dont fait l'objet ce papier. Si l'on peut vérifier et corriger la 
Tableau 1. Répartition des femmes interrogées par délégation au regard du plan d'échantillonnage.

Table 1. Distribution of women by delegation in relation with the sampling plan.

\begin{tabular}{|c|c|c|c|c|c|c|}
\hline \multirow[t]{2}{*}{ Délégation } & \multirow{2}{*}{$\begin{array}{l}\text { Plan d'échantillonnage } \\
\text { Nombre de femmes } \\
\text { à interroger }\end{array}$} & \multicolumn{5}{|c|}{ Femmes interrogées de 20 à 49 ans $(n=575)$} \\
\hline & & $n$ & $\%$ & $n$ & $\%$ & $\frac{\text { Total }}{N}$ \\
\hline Sidi Bouzid Ouest & 120 & 78 & 83,9 & 15 & 16,1 & 93 \\
\hline Sidi Bouzid Est & 80 & 0 & 0 & 67 & 100 & 67 \\
\hline Jilma & 80 & 29 & 46,8 & 33 & 53,2 & 62 \\
\hline Sidi Ali Ben Aoun & 40 & 16 & 47,1 & 18 & 52,9 & 34 \\
\hline Menzel Bouzaine & 80 & 13 & 21,7 & 47 & 78,3 & 60 \\
\hline Meknassy & 20 & 12 & 100 & 0 & 0 & 12 \\
\hline Souk Jdid & 40 & 0 & 0 & 32 & 100 & 32 \\
\hline Mezzouna & 40 & 16 & 48,5 & 17 & 51,5 & 33 \\
\hline
\end{tabular}

représentativité des femmes au sein du gouvernorat, il n'en est pas de même pour les autres unités d'observations. La méthode de pondération présentée dans la section suivante ne permet pas à l'échantillon d'être représentatif des exploitations agricoles de la région.

Dans le cadre d'étude sur cette unité d'observation, une vigilance particulière devra être apportée à ce point en dépit des difficultés de corrections qui seront rencontrées (pas de recensement agricole existant en Tunisie).

\subsection{Pondération}

Notre objectif est d'obtenir un échantillon qui assure la meilleure représentativité possible des femmes du gouvernorat.

À partir de la base des données sur la population estimée en 2010, on obtient la répartition de la population rurale et urbaine des femmes dans chacune des délégations où l'enquête a eu lieu. On a donc aussi le poids de la population totale de femmes de chaque délégation. Pour extrapoler les résultats de l'enquête à la population féminine de Sidi-Bouzid, il faudra donc que le poids de chaque délégation et la répartition de la population rurale et urbaine au sein de chaque délégation soient respectés. Pour ce faire, la pondération a été réalisée de la façon suivante:

- au sein de chaque délégation, la répartition de la population rurale et urbaine interrogée est ramenée par pondération à la répartition de la population estimée en 2010. Dans certaines délégations, il n'y a pas de population urbaine ou rurale interrogée. Dans ce cas, nous ne redressons pas la population restante (par exemple, la population rurale si il n'y a pas de population urbaine) et nous lui appliquons un coefficient de 1 ;

- ramené à la population des 575 femmes, le poids de la population féminine doit être respecté pour être
Tableau 2. Coefficient de pondération par délégation de Sidi-Bouzid. Table 2. Weighting coefficients by delegation of Sidi-Bouzid.

\begin{tabular}{lll}
\hline Délégation & $\begin{array}{l}\text { Pondération des } \\
\text { districts urbains }\end{array}$ & $\begin{array}{l}\text { Pondération des } \\
\text { districts ruraux }\end{array}$ \\
\hline Sidi Bouzid Ouest & 0,747 & 2,859 \\
Sidi Bouzid Est & Absence de population 1,007 \\
Jilma & 0,259 & 1,322 \\
Cebalit Ouled Asker Absence de population & 1,532 \\
Bir Elhfay & Absence de population 1,117 \\
Sidi Ali Ben Aoun & 0,769 & 1,389 \\
Menzel Bouzaine & 0,765 & 0,522 \\
Meknassy & 2,706 & Absence de population \\
Souk Jdid & Absence de population 0,927 \\
Mezzouna & 0,609 & 1,338 \\
Rgueb & Absence de population 0,969 \\
Ouled Haffouz & Absence de population 0,811 \\
\hline
\end{tabular}

représentatif du gouvernorat. On affecte donc à chaque délégation une pondération qui permettra de revenir à la population estimée en 2010 ;

- ces coefficients (d'un côté ceux de la répartition urbain/ rural et d'un autre celui de la délégation) sont ensuite multipliés entre eux pour obtenir le coefficient de pondération final, un coefficient par délégation et par zone rurale/urbain.

Ainsi, comme le tableau 2 l'indique, une femme qui vit dans la zone urbaine de Sidi-Bouzid-Ouest verra appliquer à ses réponses un poids de 0,747 , quand on appliquera aux réponses d'une femme vivant en milieu rural à Cebalit Ouled Asker un poids de 1,532. 


\subsection{Mode de collecte et administration des questionnaires}

Pour chacune des 3 unités d'observation citées précédemment, un questionnaire spécifique a été élaboré.

Cette enquête s'appuie sur l'échantillon de femmes constitué pour l'enquête nutritionnelle conduite en quatre passages sur une année. Les femmes constituent ainsi notre entrée principale et sont interrogées en premier. Le questionnaire permet d'identifier le ménage et son responsable, et le cas échéant, l'exploitation agricole. Notons que la femme interrogée peut être (ou non) chef de ménage, elle peut aussi être "chef d'exploitation». Cela a des conséquences sur l'organisation et la conduite du travail d'enquête.

\subsubsection{Phase pilote}

Les questionnaires d'enquête ont été finalisés dans le cadre d'une phase pilote d'une semaine auprès de 16 ménages hors échantillon en zone urbaine et rurale. Cette phase a impliqué la formation et la sélection finale des enquêtrices, le test des questionnaires et la création du masque de saisie. Elle s'est déroulée du $1^{\text {er }}$ au 8 décembre 2015.

$\mathrm{Au}$ cours de cette étape, des personnes ressources ont présenté leurs travaux, ainsi que des éléments de compréhension du contexte du gouvernorat de Sidi Bouzid.

Ces présentations ont aussi contribué à la finalisation du questionnaire. Les principales révisions des instruments de l'enquête ont concerné la définition et l'adaptation en dialecte local de questions posées dans les trois questionnaires. Des précisions ont été apportées sur les unités locales de mesure et sur les dimensions non monétaires des rémunérations et des échanges.

\subsubsection{Formation des enquêtrices}

Toutes les enquêtrices sélectionnées sont originaires de Sidi-Bouzid. La formation suivie a permis de présenter les procédures de collecte uniformisées et d'apporter des instructions précises sur toutes les questions, en particulier celles relatives aux exploitations agricoles nécessitant davantage de connaissances.

\subsection{Type d'instrument et procédure de collecte}

Compte tenu du type exploratoire et relationnel de notre étude, le choix de l'instrument de recherche s'est naturellement orienté sur la conduite de trois questionnaires directifs, structurés autour d'une majorité de réponses pré-codées et administrées en face-à-face. La première phase d'enquête s'est déroulée du 10 au 29 décembre 2015, la seconde du 4 au 21 janvier 2016, soit 36 jours effectifs d'enquête, rattrapages compris, ce qui fait une moyenne de 20 ménages par jour, soit un district par jour. L'équipe de collecte des données a été constituée de 7 enquêtrices réparties en deux groupes: les ingénieures en agronomie en charges de l'administration du questionnaire «exploitation »; et les enquêtrices de niveau bac +3 chargées de l'administration des questionnaires «ménage» et «femme». Chaque équipe de collecte a été complétée par la présence d'un superviseur, un data manager et un chauffeur.
En premier lieu, les femmes à interroger et les membres de leur ménage sont sollicités afin de leur expliquer les objectifs de l'enquête, repérer les personnes à interroger et vérifier leur disponibilité. En fonction des réponses, approbation ou non de la part des répondants, les superviseurs organisent la répartition des enquêtrices. Dès les premiers échanges, les superviseurs tentent donc de savoir si la femme est liée à une exploitation agricole familiale à l'échelle de son ménage, pour ainsi anticiper la conduite du questionnaire «exploitation» et la mobilisation en conséquence d'une enquêtrice.

Chaque femme interrogée doit être attachée à un ménage. Rappelons que le ménage est défini comme étant le groupe qui partage un seul et même budget depuis au moins 6 mois ou qui compte le faire pour au moins 6 mois. Dans la région, on utilise le terme de «kèsa», la «caisse». Il peut s'agir d'une simple famille nucléaire ou de deux frères mariés avec leur famille respective, mais qui font budget commun. De manière indirecte, il a été demandé qui est le ou la gestionnaire des dépenses, et in fine de la «kèsa» du ménage, en listant lors de la conversation d'introduction les grands postes de dépenses que cet entretien supposait. Finalement, l'individu systématiquement interrogé est celui concentrant le plus d'informations sur les dépenses du «ménage».

Pour le cas des ménages agricoles, la procédure a été la même en évitant l'appellation «chef d'exploitation», pour déceler, au gré de quelques échanges informels en amont de la conduite d'entretien, qui était véritablement l'individu du ménage concentrant le plus de connaissances sur les détails de gestion de l'exploitation. Ces échanges sur la caractérisation des administrés en amont des enquêtes furent menés par les superviseurs, munis de documents de suivi opératoire: listes des ménages par district complétées avec des informations d'ordre socio-économique.

\subsection{Caractéristiques de la collecte des données}

Les temps d'administration des différents questionnaires «Femme», «Ménage» et «Exploitation», sont respectivement en moyenne de trente minutes, dix minutes et d'environ quarante minutes.

Il a été convenu de commencer la collecte sur les terrains dont les conditions d'accessibilité et climatiques sont les plus difficiles. L'équipe de collecte a donc débuté par les zones montagneuses au nord-ouest du gouvernorat, pour profiter du climat clément à cette période de l'enquête. Les enquêtes se font généralement en extérieur, près des maisonnées.

L'administration des questionnaires dans les zones urbaines a été facilitée par une meilleure accessibilité, par rapport aux maisonnées dispersées caractéristiques des zones rurales isolées.

\subsection{Saisie des questionnaires}

Le masque de saisie a été créé en amont de la collecte, une fois le questionnaire finalisé.

Vingt-sept tables liées par la clé primaire, identifiant unique du ménage, ont été construites et organisées pour correspondre aux unités d'observation.

Une formation de quatre jours a été organisée pour les agents de saisie. Le logiciel Epidata permet de réaliser une 
double saisie, fonctionnalité qui rend possible la confrontation de deux saisies d'un même questionnaire administré. Le choix du logiciel a été guidé par cette fonctionnalité minimisant le taux d'erreur de saisie.

\subsection{Nettoyage des données}

À l'issue de l'export des données sur Stata, les données ont été transformées afin d'obtenir, en partant des 27 tableaux initiaux, 3 fichiers correspondant aux 3 unités d'observation et un fichier unique qui a permis d'explorer les liens entre toutes les variables disponibles. Par ailleurs, les 27 fichiers sont également conservés pour faire des analyses spécifiques par thématique.

Plusieurs types de contrôle ont été effectués :

- la cohérence des réponses liées (ex: surface irriguée > surface totale);

- la gestion des non-réponses matérialisées par un «.» dans Stata et différentes de 0 ;

- le contrôle des valeurs quantitatives extrêmes (la médiane $+/-3$ fois l'écart-type).

Certaines valeurs contrôlées ont fait l'objet de vérification de questionnaire ou même parfois d'enquêtes téléphoniques complémentaires.

On notera un taux de non-réponse marginale au sein des questionnaires récoltés. Ces non-réponses peuvent concerner des questions très précises sur les dépenses par type de produits, les sous totaux par type étant très majoritairement renseignés.

\subsection{Anonymisation des données}

Les données récoltées à Sidi-Bouzid présentent quelques caractéristiques pouvant être considérées comme sensibles. Ainsi, la perception de la sécurité alimentaire des femmes, le niveau de dépenses des ménages ou les revenus générés par les exploitations agricoles sont des informations qui doivent rester anonymes.

Dans l'objectif de conserver un potentiel élevé de réutilisation des données et afin de préserver l'anonymat des personnes interrogées, les techniques de pseudonymisation et de k-anonymisation ont été utilisées. Ces techniques permettent, à travers la reconstruction de certaines variables et la suppression d'autres, de se protéger contre les attaques appelées «records linkage».

L'objectif de la k-anonymisation est d'obtenir un fichier dont les caractéristiques sensibles concernent au moins $k$ individus (avec $k>1$ ). Une première pseudonymisation a été effectuée sur les fichiers en retirant de l'étude les variables d'identification. Les variables dites de pseudo-identification, permettant de repérer un individu par des caractéristiques atypiques ou la combinaison de caractéristiques, ont ensuite fait l'objet de traitement (recodage par tranche, agrégation de données...). Le grand nombre de variables ne permet pas d'arriver à k-anonymiser la base sur toutes les variables pseudo-identifiantes et sensibles du fichier. Cependant, en utilisant cette méthode, les regroupements effectués permettent de garder à la fois un anonymat (plus de deux occurrences) sur deux variables prises 2 à 2 (une variable de pseudo- identification et une variable sensible) et de conserver un bon niveau d'information.

Ainsi on a supprimé les variables d'identification suivantes :

- nom, prénom de la personne interrogée (femme, chef de ménage et chef de l'exploitation agricole);

- lien de parenté potentiel entre les différents niveaux d'observation;

- statut matrimonial de la femme interrogée;

- statut juridique de l'exploitation;

- coordonnées GPS de l'exploitation;

- année de création ou d'achat de l'exploitation.

Les variables quantitatives de pseudo-identification (en cas de valeurs extrêmes) recodées en tranche sont les suivantes:

- surface de l'exploitation;

- nombre d'enfants à charge.

La variable qualitative «activité agricole principale» est également une variable de pseudo-identification sur laquelle on crée des regroupements.

Enfin des variables jugées sensibles ont été recodées par tranche ou ont été agrégées en score (IFIAS) :

- revenus monétaires individuels des personnes interrogées ;

- revenus monétaires issus de la production agricole;

- revenus monétaires distribués à la main-d'œuvre extérieure ;

- dépenses du ménage par grands sous-types;

- sécurité alimentaire de la femme (IFIAS).

Par ailleurs, les dépenses détaillées du ménage, les effectifs détaillés du cheptel et les réponses au questionnaire IFIAS ont été agrégés. Les variables qui ne présentaient pas un intérêt fondamental dans l'analyse et dont la fréquence de certaines modalités est très faible, comme par exemple le statut juridique, ont été retirées des fichiers.

\section{Description des fichiers de données}

Les fichiers de données sont décrits dans le tableau 3.

Tableau 3. Description des fichiers de données. Table 3. Description of data files.

Nombre total de fichiers disponibles contenant des données associées à l'étude

Type de fichiers (ASCII, EBCDIC, etc.) et

logiciel associé comme les «fichiers de données SAS », les «fichiers export SPSS », etc.

Logiciel(s) utilisé(s) pour produire le fichier et version du logiciel

Nombre total d'observations par fichier

EPIDATA 3.1 /STATA 14

STATA

Fichier Femme : 575

Fichier Ménage : 575

Fichier Exploitation agricole : 316

Nombre total de variables par fichier

Fichier Ménage : 22

Fichier Exploitation

agricole : 163 


\section{Localisation et conditions d'accès aux données}

Les données sont disponibles sur le lien doi: 10.18167/ DVN1/LWT7BG. Un embargo de 1 an est prévu avant l'accès aux fichiers de données.

\section{Potentiel de réutilisation des données}

Cette étude a été menée dans une période où le contexte était difficile pour les populations locales. Les changements associés aux évènements de fin 2010 ont été importants. Les nouvelles politiques en place sont donc susceptibles de faire évoluer le pays et la région. Des comparaisons avec des études similaires futures auront donc un intérêt particulier. Des changements de perception de la sécurité alimentaire chez les femmes aux analyses des transformations agricoles, de nombreuses questions de recherche peuvent être menées à partir de la comparaison avec l'étude présente.

La Tunisie devrait mener au cours de l'année 2018 le premier recensement agricole de son histoire. Ce recensement serait complété par une enquête communautaire réalisée auprès des ménages. Les informations de la région de SidiBouzid pourront aussi être comparées avec les informations exhaustives recueillies à l'issue de ces enquêtes.

De façon générale, les questions de recherche soulevées dans cette étude sont communes à toute la zone méditerranéenne. Des études comparatives sur d'autres terrains d'étude de la zone pourraient avoir un intérêt.

Enfin, des enquêtes plus ciblées sur la nutrition pourraient utiliser ces données dans le cadre de comparaisons entre les niveaux de consommation alimentaire et les achats des ménages ou les niveaux de perception d'insécurité alimentaire.

Une mise en commun des résultats est déjà prévue avec les données de consommation alimentaire collectées lors des quatre premiers passages par l'IRD et l'INNTA.

Remerciements. Cette enquête doit beaucoup au travail des équipes d'enquêtrices et d'agents de saisie engagés et encadrés par les services de l'INNTA. En particulier, les appuis et soutiens sur le terrain de Zayneb Dahbi, coordinatrice du volet agricole et du data management, ainsi que ceux de Wafa Tlili, ingénieur développement local de la Gesellschaft für Internationale Zusammenarbeit (GIZ), ont largement contribué au bon déroulement de l'enquête. Cette enquête est aussi le fruit d'un travail collaboratif du Groupe d'études du projet MEDINA, comprenant, outre les auteurs de l'article: MJ Amiot (INRA), N Achir (CIRAD), L Alouane (INNTA), S Bellagha (INAT), M Broin (Agropolis), N Darmon (INRA), C Dhuique-Meyer (CIRAD), S Drogué (INRA), A Ferchoui (INRA), Z Ghrabi (INAT), F Jacquet (INRA), Y Kameli (IRD), F Kefi (CIHEAM), E Kesse-Guyot (INRA), D Lairon (INSERM), Y Martin-Prevel (IRD), C Méjean (INRA), C Mouquet (IRD), S Njoumi (INAT), M Padilla (CIHEAM), M Pérignon (INRA), C Sinfort (SupAgro), P Traissac (IRD), E Verger (IRD).

\section{Références}

Alkire S, Meinzen-Dick R, Peterman A, Quisumbing AR, Seymour G, Vaz A. 2013. The women's empowerment in agriculture index. World Development 52: 71-91. DOI: 10.1016/j.world dev.2013.06.007.

Carletto G, Ruel M, Winters P, Zezza A. 2015. Farm-level pathways to improved nutritional status: introduction to the special issue. The Journal of Development Studies 51(8): 945-957. DOI: 10.1080/ 00220388.2015.1018908.

Coates J, Swindale A, Bilinsky P. 2007. Household Food Insecurity Access Scale (HFIAS) for Measurement of Household Food Access: Indicator Guide (v.3). Washington, D.C.: FHI 360/ FANTA. https://www.fantaproject.org/monitoring-and-evaluation/ household-food-insecurity-access-scale-hfias.

CREDIF. 2003. Femmes et développement régional en Tunisie, tome I, Tunis, CREDIF, 216 p.

FAO AQUASTAT. 2014. Rôle des femmes dans la gestion des ressources en eau en général et de l'eau agricole en particulier. Rapport du projet pilote de développement d'indicateurs sensibles au genre et leur intégration potentielle dans AQUASTAT, le système mondial d'information sur l'eau. Rapport, 154 p. Disponible sur http://www.fao.org/3/a-bc820f.pdf.

Ferchiou A. 2017. Quelles mesures de relances pour l'agriculture familiale en zone aride : Évaluation intégrée par la modélisation bio économique des ménages agricoles de Sidi Bouzid. Thèse, Institut national d'études supérieures agronomiques de Montpellier.

Gasmi M. 2003. Accés des femmes rurales à la terre en Tunisie entre le droit et le vécu. Exposé à l'atelier international «Femmes rurales et foncier », projet FAO-Dimitra. Disponible sur http://www.fao. org/fileadmin/templates/dimitra/pdf/thies2003_tunisie.pdf.

Gillespie S, Kadiyala S. 2012. Exploring the agriculture nutrition disconnect in India. In: Leveraging Agriculture for Improving Nutrition and Health 2020 Conference book. Washington DC: International Food Policy Research Institute (IFPRI), pp. 173-181.

Gillespie S, Jody H, Suneetha K. 2012. The agriculture-nutrition disconnect in India: What do we know? IFPRI discussion papers 1187. Washington DC: International Food Policy Research Institute (IFPRI).

Gomez MI, Barrett CB, Raney T, Pinstrup-Andersen P, Meerman J, Croppenstedt A, et al. 2013. Post-green revolution food systems and the triple burden of malnutrition. Food Policy 42: 129-138. DOI: 10.1016/j.foodpol.2013.06.009.

Haddad L. 2000. A conceptual framework for assessing agriculturenutrition linkages. Food and Nutrition Bulletin 21(4): 367-373. DOI: $10.1177 / 156482650002100405$.

Herforth A, Lidder P, Gill M. 2015. Strengthening the links between nutrition and health outcomes and agricultural research. Food Security 7(3): 457-461. DOI: 10.1007/s12571-015-0451-z.

Hoddinott J. 2012. Agriculture, health, and nutrition: Toward conceptualizing the linkages. In: Fan S, Pandya-Lorch R, eds. Reshaping agriculture for nutrition and health. Washington, DC: International Food Policy Research Institute. DOI: 10.2499/ 9780896296725 .

Institut national de la statistique (INS). 2014. Recensement général de la population et de l'habitat. Disponible sur http://census.ins.tn/ recensement.

Latreille M. 2008. Honor, the gender division of labor, and the status of women in rural Tunisia - a social organizational reading. Middle East Stud 40: 599-621. DOI: 10.1017/S0020743808081518. 
Malapit HJL, Kadiyala S, Quisumbing AR, Cunningham K, Tyagi P. 2015. Women's empowerment mitigates the negative effects of low production diversity on maternal and child nutrition in Nepal. The Journal of Development Studies 51(8): 1097-1123. DOI: 10.1080/ 00220388.2015.1018904.

Martin-Prével Y. 2002. "Soins» et nutrition publique. Cahiers d'Études et de Recherches Francophones/Santé 12(1): 86-93.
ONU. 2010. Guide pratique pour la conception d'enquêtes sur les ménages, Département des affaires économiques et sociales, division statistique.

Sibhatu KT, Krishna VV, Qaim M. 2015. Production diversity and dietary diversity in smallholder farm households. Proc Natl Acad Sci USA 112: 10657-10662. DOI: 10.1073/pnas. 1510982112.

Citation de l'article : Gaillard C, Martin S, Bosc P-M, El-Ati J, Dop MC, Trabelsi T, Amiot M-J, Dury S. 2018. Explorer les liens entre agriculture et sécurité alimentaire: une enquête auprès des femmes du gouvernorat de Sidi-Bouzid en Tunisie. Cah. Agric. 27: 15501. 